\title{
Supporting information for Conformational Mobility and Proton Transfer in Hydrogen-Bonded Dimers and Trimers of Phosphinic and Phosphoric Acids
}

\author{
Valeriya V. Mulloyarova, ${ }^{\dagger}$ Ivan S. Giba, ${ }^{+\ldots}+$ Gleb S. Denisov, ${ }^{\ddagger}$ Alexei S. Ostras’† and Peter M. \\ Tolstoy ${ }^{*}$, \\ †Institute of Chemistry, St.Petersburg State University, Universitetskij pr. 26, 198504 St. Petersburg, Russia \\ ‡ Department of Physics, St.Petersburg State University, Ulyanovskaya 1, 198504, St. Petersburg, Russia \\ * - corresponding author, peter.tolstoy@spbu.ru
}

\section{Contents}

Table S1 Calculated energetic, geometric and spectral parameters for monomers, cyclic dimers and cyclic trimers of phosphinic and phosphoric acids $\mathbf{1 - 5}$.

Table S2 Hydrogen bond energies, selected equilibrium geometric and spectral parameters for monomers and cyclic dimers of hypophosphinic acid $\mathbf{1}$ and dimethylphopshinic acid $\mathbf{2}$, calculated at various levels of theory and various basis sets.

Table S3 Calculated distances from phosphorous atom to its immediate neighbors in monomers, cyclic dimers and cyclic trimers of acids $\mathbf{1}-\mathbf{5}$ in vacuum.

Figure S1 Calculated equilibrium structures of monomer, cyclic dimer and cyclic trimer of dimethylphosphinic acid $\mathbf{2}$ in vacuum.

Figure S2 Calculated equilibrium structures of dimer and cyclic trimer of phenylphosphinic acid 3 in vacuum.

Figure S3 Calculated equilibrium structures of cyclic dimer of dimethylphosphoric acid 4 in vacuum.

Figure S4 Calculated equilibrium structures of cyclic trimer of dimethylphosphoric acid 4 in vacuum.

Figure S5 Calculated equilibrium structure of cyclic trimer of diphenylphosphoric acid $\mathbf{5}$ in vacuum.

Figure S6 Molecular structures and energy barriers for the proton transfer and hydrogen bond ring puckering of cyclic dimer of dimethylphosphinic acid 2.

Figure S7 Calculated energy and geometry changes along the proton transfer pathway for acid 1. The case when one proton is being transferred and the other one follows.

Figure S8 Calculated energy and geometry changes along the proton transfer pathway for acid $\mathbf{1}$. The case when both protons are being transferred at the same time (forced symmetry).

Figure S9 Molecular structures and energy barriers for the proton transfer in cyclic dimer of dimethylphosphoric acid 4.

Figure S10 Molecular structures and energy barrier for the proton transfer in cyclic dimer of diphenylphosphoric acid $\mathbf{5}$.

Figure S11 The interdependence of the OPO angle and OPOH dihedral angle for cyclic dimers and trimers of acids $\mathbf{1}-\mathbf{5}$.

Figure S12 The dependence of the ${ }^{31} \mathrm{P}$ NMR chemical shift in cyclic dimers and cyclic trimers of acids 1-5 on $r_{\mathrm{OH}}$ and $r_{\mathrm{H} \cdots \mathrm{O}}$ distances.

Figure S13 Interdependence of $q_{2}=r_{\mathrm{OH}}+r_{\mathrm{H} \cdots \mathrm{O}}$ and $q_{1}=1 / 2 \cdot\left(r_{\mathrm{OH}}-r_{\mathrm{H} \cdots \mathrm{O}}\right)$ distances for acids $\mathbf{1}-\mathbf{5}$.

Figure $\mathrm{S} 14 \quad$ Interdependence of $\mathrm{P}-\mathrm{OH}$ and $\mathrm{P}=\mathrm{O}$ distances in cyclic dimers and cyclic trimers of acids 1-5 and the sensitivity of $\delta \mathrm{P}$ to these distances. 
Table S1. Calculated (B3LYP/6-311++(d,p)) energetic (BSSE corrected), geometric and spectral parameters for monomers, cyclic dimers and cyclic trimers of phosphinic and phosphoric acids 1-5. The relative energy $\left(\Delta E_{\text {rel }}\right)$ for each set of complexes of acids 1-5 was calculated with respect to the most stable conformer. The energy of hydrogen bond $\left(\Delta E_{\mathrm{HB}}\right)$ was obtained per one H-bond. The energy of transition state $\left(\Delta E_{\mathrm{TS}}\right)$ for concerted multiple proton transfer was calculated relative to the more stable form. All energies are given in $\mathrm{kcal} / \mathrm{mol}$. The $\mathrm{O}-\mathrm{H}$ bond length $(r(\mathrm{OH}))$ and the distance from bridging proton to $\mathrm{P}=\mathrm{O}$ oxygen $(r(\mathrm{H} \cdots \mathrm{O}))$ are given in $\AA$; the hydrogen bond angle $(\angle \mathrm{OHO})$ and the dihedral angle $(\angle \mathrm{OPOH})-$ in degrees. The ${ }^{1} \mathrm{H}$ and ${ }^{31} \mathrm{P}$ NMR chemical shifts $(\delta \mathrm{H}$ and $\delta \mathrm{P})$ are given in ppm.

\begin{tabular}{|c|c|c|c|c|c|c|c|c|c|c|c|}
\hline Complex & Figure & $\begin{array}{c}\Delta E_{\text {rel }}, \\
\mathrm{kcal} / \mathrm{mol}\end{array}$ & $\begin{array}{c}\Delta E_{\mathrm{HB}}{ }^{\mathrm{a}} \\
\mathrm{kcal} / \mathrm{mol}\end{array}$ & $\begin{array}{c}\Delta E_{\mathrm{TS}}, \\
\mathrm{kcal} / \mathrm{mol}\end{array}$ & $\begin{array}{c}r(\mathrm{OH}), \\
\AA\end{array}$ & $\begin{array}{c}r(\mathrm{H} \cdots \mathrm{O}), \\
\AA\end{array}$ & $\underset{\circ}{\angle \mathrm{OHO},}$ & $\begin{array}{l}\angle \mathrm{OPO} \\
\mathrm{H},{ }^{\mathrm{b}}{ }^{\circ}\end{array}$ & $\angle \mathrm{OPO},^{\circ}$ & $\delta \mathrm{H}, \mathrm{ppm}$ & $\delta \mathrm{P}, \mathrm{ppm}$ \\
\hline $1 \mathrm{M}$ & $2 a$ & 0 & - & & 0.965 & - & - & 39.9 & 117.6 & 3.45 & -16.80 \\
\hline \multirow[t]{2}{*}{ 1D-a } & $2 b$ & 0 & 11.3 & 5.3 & 1.005 & 1.623 & 166.3 & 40.2 & 117.2 & 11.49 & -9.60 \\
\hline & & & & & 1.005 & 1.622 & 166.3 & 40.3 & 117.2 & 11.49 & -9.60 \\
\hline \multirow[t]{2}{*}{ 1D-b } & $2 b$ & +0.4 & 11.1 & 5.8 & 1.004 & 1.627 & 166.4 & 38.0 & 117.4 & 11.31 & -9.07 \\
\hline & & & & & 1.004 & 1.629 & 166.4 & 38.0 & 117.4 & 11.29 & -9.09 \\
\hline \multirow[t]{3}{*}{ 1T-a } & $2 c$ & 0 & 13.8 & 5.7 & 1.013 & 1.535 & 173.7 & 66.1 & 118.9 & 13.50 & -19.46 \\
\hline & & & & & 1.014 & 1.530 & 175.2 & 63.8 & 119.0 & 13.51 & -17.80 \\
\hline & & & & & 1.014 & 1.530 & 175.5 & 68.7 & 118.9 & 13.25 & -19.01 \\
\hline \multirow[t]{3}{*}{ 1T-b } & $2 \mathrm{c}$ & +0.3 & 13.8 & & 1.013 & 1.538 & 173.9 & 63.8 & 119.2 & 13.21 & -18.12 \\
\hline & & & & & 1.013 & 1.537 & 173.9 & 63.7 & 119.1 & 13.23 & -18.21 \\
\hline & & & & & 1.012 & 1.538 & 173.8 & 64.2 & 119.2 & 13.22 & -18.20 \\
\hline $2 M$ & S1a & 0 & - & & 0.965 & - & - & 0.1 & 113.4 & 2.77 & 38.42 \\
\hline \multirow[t]{2}{*}{ 2D-a } & S1b & 0 & 11.4 & 4.6 & 1.009 & 1.594 & 171.0 & 36.0 & 114.9 & 11.67 & 39.29 \\
\hline & & & & & 1.009 & 1.594 & 171.0 & 35.9 & 114.9 & 11.67 & 39.29 \\
\hline \multirow[t]{2}{*}{ 2D-b } & S1b & +0.3 & 11.3 & 4.7 & 1.011 & 1.579 & 173.0 & 23.9 & 115.0 & 11.78 & 39.69 \\
\hline & & & & & 1.011 & 1.579 & 173.0 & 24.0 & 115.0 & 11.78 & 39.69 \\
\hline \multirow[t]{3}{*}{$2 \mathrm{~T}-\mathrm{a}$} & S1c & 0 & 13.2 & 5.5 & 1.014 & 1.541 & 173.6 & 67.7 & 116.2 & 12.84 & 31.27 \\
\hline & & & & & 1.016 & 1.534 & 174.5 & 65.7 & 116.3 & 12.71 & 32.49 \\
\hline & & & & & 1.014 & 1.541 & 174.0 & 72.8 & 116.1 & 12.97 & 31.43 \\
\hline \multirow[t]{3}{*}{ 2T-b } & S1c & +0.4 & 13.1 & & 1.015 & 1.539 & 174.4 & 63.7 & 116.6 & 12.73 & 32.13 \\
\hline & & & & & 1.015 & 1.540 & 173.9 & 63.6 & 116.5 & 12.77 & 32.38 \\
\hline & & & & & 1.015 & 1.540 & 174.3 & 63.9 & 116.5 & 12.75 & 32.32 \\
\hline $3 M$ & $3 a$ & 0 & - & & 0.965 & - & - & 41.0 & 116.7 & 3.61 & 1.30 \\
\hline \multirow[t]{2}{*}{ 3D-a } & $3 b$ & +0.4 & 11.0 & 5.0 & 1.009 & 1.599 & 170.4 & -34.2 & 116.9 & 11.83 & 7.57 \\
\hline & & & & & 1.009 & 1.598 & 170.4 & -34.1 & 116.9 & 11.83 & 7.59 \\
\hline \multirow[t]{2}{*}{ 3D-b } & $3 b$ & +0.2 & 11.3 & 4.6 & 1.010 & 1.598 & 169.8 & 37.7 & 116.9 & 12.16 & 8.16 \\
\hline & & & & & 1.008 & 1.600 & 169.5 & -35.3 & 116.8 & 12.23 & 4.34 \\
\hline \multirow[t]{2}{*}{ 3D-c } & $3 b$ & 0 & 11.6 & 4.6 & 1.009 & 1.600 & 168.9 & -38.5 & 116.8 & 12.44 & 4.16 \\
\hline & & & & & 1.009 & 1.600 & 168.9 & -38.5 & 116.8 & 12.45 & 4.14 \\
\hline \multirow[t]{2}{*}{ 3D-d } & S2a & +0.3 & 11.2 & 4.6 & 1.009 & 1.596 & 170.4 & 39.3 & 116.9 & 11.95 & 8.87 \\
\hline & & & & & 1.007 & 1.606 & 168.6 & -30.6 & 116.9 & 12.13 & 4.72 \\
\hline \multirow[t]{2}{*}{ 3D-e } & S2a & +0.3 & 10.9 & & 1.008 & 1.601 & 169.7 & -34.4 & 116.9 & 11.79 & 8.03 \\
\hline & & & & & 1.008 & 1.601 & 169.7 & -34.3 & 116.9 & 11.79 & 8.03 \\
\hline \multirow[t]{2}{*}{ 3D-f } & S2a & +0.3 & 11.4 & & 1.008 & 1.599 & 169.5 & 35.0 & 116.9 & 12.23 & 4.56 \\
\hline & & & & & 1.008 & 1.599 & 169.5 & 35.0 & 116.9 & 12.24 & 4.56 \\
\hline \multirow[t]{3}{*}{ 3T-a } & 4 & +0.3 & 13.7 & & 1.014 & 1.534 & 174.9 & 69.3 & 118.7 & 13.62 & -2.07 \\
\hline & & & & & 1.014 & 1.535 & 175.1 & 68.7 & 118.7 & 13.70 & -4.68 \\
\hline & & & & & 1.014 & 1.535 & 174.8 & 68.2 & 118.6 & 13.69 & -2.64 \\
\hline \multirow[t]{3}{*}{$3 T-b$} & 4 & +0.6 & 13.4 & & 1.016 & 1.528 & 177.8 & -58.0 & 118.6 & 13.37 & 1.15 \\
\hline & & & & & 1.015 & 1.530 & 175.3 & 68.7 & 118.7 & 14.04 & -4.55 \\
\hline & & & & & 1.013 & 1.539 & 175.1 & 70.4 & 118.8 & 13.51 & -3.65 \\
\hline 3T-c & 4 & +0.8 & 13.2 & & 1.013 & 1.538 & 174.5 & 72.2 & 118.8 & 13.68 & -5.80 \\
\hline & & & & & 1.015 & 1.533 & 177.6 & -60.3 & 118.6 & 13.20 & -0.89 \\
\hline & & & & & 1.018 & 1.523 & 178.2 & -57.3 & 118.7 & 13.59 & 0.14 \\
\hline 3T-d & 4 & $\begin{array}{l}+1.0 \\
\end{array}$ & 12.9 & & 1.015 & 1.541 & 177.6 & -61.1 & 118.8 & 13.13 & -0.61 \\
\hline & & & & & 1.013 & 1.538 & 177.3 & -62.7 & 118.9 & 13.23 & -0.82 \\
\hline & & & & & 1.014 & 1.534 & 177.7 & -62.2 & 118.7 & 13.22 & -1.43 \\
\hline 3T-e & $\mathrm{S} 2 \mathrm{~b}$ & 0 & 13.8 & 5.3 & 1.016 & 1.525 & 176.0 & 69.5 & 118.5 & 14.24 & -3.11 \\
\hline & & & & & 1.014 & 1.532 & 174.8 & 70.6 & 118.4 & 13.75 & -4.48 \\
\hline & & & & & 1.017 & 1.524 & 176.0 & 74.6 & 118.4 & 14.24 & -5.19 \\
\hline 3T-f & $\mathrm{S} 2 \mathrm{~b}$ & +0.3 & 13.5 & & 1.019 & 1.519 & 177.9 & -59.3 & 118.5 & 13.83 & 0.82 \\
\hline & & & & & 1.015 & 1.529 & 174.9 & 70.0 & 118.5 & 13.98 & -5.04 \\
\hline & & & & & 1.016 & 1.527 & 175.9 & 74.8 & 118.5 & 14.09 & -5.85 \\
\hline $3 T-g$ & $\mathrm{~S} 2 \mathrm{~b}$ & +0.5 & 13.4 & & 1.015 & 1.529 & 176.0 & 71.0 & 118.5 & 13.40 & -4.68 \\
\hline
\end{tabular}




\begin{tabular}{|c|c|c|c|c|c|c|c|c|c|c|c|}
\hline & & & & & 1.016 & 1.5288 & 177.9 & -62.6 & 118.4 & 14.01 & 0.45 \\
\hline & & & & & 1.015 & 1.527 & 175.8 & 75.5 & 118.3 & 14.02 & -5.45 \\
\hline \multirow[t]{3}{*}{ 3T-h } & $\mathrm{S} 2 \mathrm{~b}$ & +0.6 & 13.4 & & 1.015 & 1.527 & 176.1 & 70.2 & 118.5 & 13.94 & -3.34 \\
\hline & & & & & 1.013 & 1.537 & 174.7 & 71.1 & 118.6 & 13.47 & -5.69 \\
\hline & & & & & 1.018 & 1.522 & 177.2 & -65.5 & 118.4 & 13.83 & 0.05 \\
\hline \multirow{3}{*}{ 3T-i } & $\mathrm{S} 2 \mathrm{~b}$ & +0.7 & 13.2 & & 1.017 & 1.526 & 177.9 & -60.9 & 118.5 & 13.50 & 0.49 \\
\hline & & & & & 1.017 & 1.526 & 177.3 & -63.2 & 118.5 & 13.63 & -0.53 \\
\hline & & & & & 1.013 & 1.533 & 175.8 & 76.0 & 118.5 & 13.63 & -6.08 \\
\hline \multirow[t]{3}{*}{$3 \mathrm{~T}-\mathrm{j}$} & $\mathrm{S} 2 \mathrm{~b}$ & +0.8 & 13.2 & & 1.017 & 1.520 & 177.1 & -60.0 & 118.5 & 13.66 & 0.25 \\
\hline & & & & & 1.015 & 1.533 & 174.9 & 70.6 & 118.6 & 13.72 & -5.78 \\
\hline & & & & & 1.017 & 1.525 & 177.2 & -65.2 & 118.5 & 13.64 & -0.97 \\
\hline \multirow[t]{3}{*}{ 3T-k } & $\mathrm{S} 2 \mathrm{~b}$ & +1.0 & 13.1 & & 1.014 & 1.532 & 176.1 & 71.4 & 118.5 & 13.75 & -4.30 \\
\hline & & & & & 1.015 & 1.533 & 177.8 & -62.6 & 118.5 & 13.10 & 0.92 \\
\hline & & & & & 1.016 & 1.525 & 176.4 & -66.7 & 118.4 & 13.64 & 0.16 \\
\hline \multirow[t]{3}{*}{ 3T-I } & $\mathrm{S} 2 \mathrm{~b}$ & +1.1 & 12.9 & & 1.015 & 1.528 & 177.3 & -61.8 & 118.5 & 13.32 & -0.63 \\
\hline & & & & & 1.015 & 1.531 & 177.4 & -63.3 & 118.6 & 13.27 & -1.11 \\
\hline & & & & & 1.014 & 1.532 & 176.2 & -67.5 & 118.5 & 13.32 & -0.70 \\
\hline $4 \mathrm{M}-\mathrm{a}$ & $5 a$ & 0 & - & & 0.965 & - & - & 26.8 & 114.8 & 3.56 & -0.89 \\
\hline $4 M-b$ & $5 a$ & +0.3 & - & & 0.965 & - & - & 21.3 & 113.7 & 3.25 & -9.14 \\
\hline 4M-c & $5 a$ & +1.1 & - & & 0.965 & - & - & 0.1 & 111.7 & 3.46 & 0.15 \\
\hline 4M-d & $5 a$ & +1.2 & - & & 0.964 & - & - & 53.1 & 118.0 & 3.52 & -8.71 \\
\hline \multirow[t]{2}{*}{ 4D-aa } & S3 & $\begin{array}{l}+0.3 \\
\end{array}$ & 11.0 & & 1.010 & 1.580 & 173.3 & 24.9 & 115.5 & 11.89 & -2.01 \\
\hline & & & & & 1.010 & 1.580 & 173.3 & 24.8 & 115.4 & 11.89 & -2.02 \\
\hline \multirow[t]{2}{*}{ 4D-ab } & $5 b$ & $\begin{array}{l}+0.3 \\
\end{array}$ & 11.1 & & 1.010 & 1.577 & 174.1 & 21.9 & 114.9 & 11.62 & -7.30 \\
\hline & & & & & 1.009 & 1.580 & 173.0 & 21.4 & 115.5 & 11.92 & -1.84 \\
\hline \multirow[t]{2}{*}{ 4D-ac } & S3 & +1.5 & 11.0 & & 1.008 & 1.583 & 175.7 & 7.5 & 113.4 & 11.73 & -2.70 \\
\hline & & & & & 1.011 & 1.567 & 173.7 & 22.2 & 115.2 & 12.17 & -1.24 \\
\hline \multirow[t]{2}{*}{ 4D-ad } & S3 & +1.2 & 11.0 & & 1.013 & 1.562 & 172.6 & 18.3 & 117.5 & 12.29 & -5.28 \\
\hline & & & & & 1.008 & 1.585 & 172.6 & 25.4 & 115.7 & 11.76 & -2.21 \\
\hline \multirow[t]{2}{*}{ 4D-bb } & $5 b$ & 0 & 11.5 & 4.8 & 1.009 & 1.581 & 173.4 & 25.3 & 115.0 & 11.59 & -6.79 \\
\hline & & & & & 1.009 & 1.581 & 173.4 & 25.3 & 115.0 & 11.59 & -6.79 \\
\hline 4D-bc & $5 b$ & +1.3 & 11.2 & & 1.011 & 1.570 & 173.0 & 27.6 & 114.8 & 11.80 & -5.97 \\
\hline & & & & & 1.007 & 1.587 & 175.3 & 12.1 & 113.3 & 11.68 & -1.86 \\
\hline 4D-bd & $5 b$ & +1.1 & 11.3 & & 1.008 & 1.587 & 172.6 & 26.8 & 115.2 & 11.42 & -6.93 \\
\hline & & & & & 1.012 & 1.565 & 171.7 & 19.8 & 117.5 & 12.27 & -5.01 \\
\hline 4D-cc & S3 & +3.0 & 10.8 & & 1.008 & 1.579 & 175.5 & 10.3 & 113.2 & 11.86 & -1.79 \\
\hline & & & & & 1.008 & 1.579 & 175.5 & 10.6 & 113.2 & 11.86 & -1.78 \\
\hline 4D-cd & S3 & +2.4 & 11.0 & & 1.006 & 1.592 & 175.5 & 7.9 & 113.4 & 11.52 & -2.30 \\
\hline & & & & & 1.014 & 1.551 & 172.0 & 21.3 & 117.3 & 12.52 & -4.54 \\
\hline 4D-dd & S3 & +2.1 & 11.1 & & 1.010 & 1.574 & 170.7 & 23.1 & 117.6 & 12.01 & -4.99 \\
\hline & & & & & 1.010 & 1.574 & 170.7 & 23.1 & 117.6 & 12.00 & -4.99 \\
\hline 4T-aаa & S4 & +0.7 & 11.8 & & 1.014 & 1.525 & 174.9 & 36.3 & 116.8 & 12.77 & -8.43 \\
\hline & & & & & 1.013 & 1.530 & 176.0 & 43.2 & 116.6 & 12.62 & -7.99 \\
\hline & & & & & 1.013 & 1.529 & 176.8 & 43.0 & 116.5 & 12.63 & -7.42 \\
\hline 4T-aab & S4 & +0.3 & 12.0 & & 1.017 & 1.515 & 175.2 & 46.6 & 117.2 & 13.08 & -8.19 \\
\hline & & & & & 1.013 & 1.533 & 175.4 & 36.9 & 115.7 & 12.31 & -10.39 \\
\hline & & & & & 1.012 & 1.537 & 178.9 & 31.5 & 116.4 & 12.43 & -6.71 \\
\hline 4T-aac & S4 & +1.1 & 11.8 & & 1.013 & 1.547 & 176.6 & 61.4 & 114.4 & 12.56 & -6.90 \\
\hline & & & & & 1.009 & 1.554 & 175.6 & 25.9 & 113.7 & 12.09 & -3.45 \\
\hline & & & & & 1.018 & 1.519 & 178.7 & 25.9 & 115.5 & 12.94 & -2.82 \\
\hline 4T-aad & S4 & +1.0 & 12.1 & & 1.015 & 1.525 & 175.9 & 42.9 & 116.9 & 12.75 & -6.89 \\
\hline & & & & & 1.015 & 1.521 & 176.3 & 54.6 & 118.8 & 12.93 & -13.03 \\
\hline & & & & & 1.011 & 1.538 & 178.7 & 42.5 & 116.2 & 12.44 & -13.03 \\
\hline 4T-bba & S4 & $\begin{array}{l}+1.1 \\
\end{array}$ & 11.8 & & 1.007 & 1.566 & 173.2 & 45.0 & 115.6 & 12.03 & -10.90 \\
\hline & & & & & 1.021 & 1.501 & 176.4 & 6.0 & 116.2 & 13.35 & -5.97 \\
\hline & & & & & 1.014 & 1.536 & 175.1 & 24.2 & 115.9 & 12.57 & -11.59 \\
\hline 4T-bbb & S4 & 0 & 12.2 & & 1.008 & 1.563 & 172.6 & 50.9 & 115.7 & 12.20 & -11.01 \\
\hline & & & & & 1.021 & 1.501 & 176.4 & 37.0 & 115.7 & 13.06 & -7.31 \\
\hline & & & & & 1.011 & 1.556 & 178.2 & 46.4 & 116.0 & 12.02 & -10.81 \\
\hline 4T-bbc & S4 & +1.8 & 11.9 & & 1.004 & 1.576 & 173.0 & 52.3 & 115.6 & 11.75 & -10.25 \\
\hline & & & & & 1.013 & 1.534 & 171.4 & 40.3 & 113.8 & 12.45 & -1.81 \\
\hline & & & & & 1.016 & 1.535 & 178.8 & 44.6 & 116.0 & 12.45 & -11.96 \\
\hline 4T-bbd & S4 & +1.1 & 12.1 & & 1.080 & 1.561 & 172.5 & 49.2 & 115.4 & 12.18 & -9.74 \\
\hline & & & & & 1.025 & 1.485 & 176.1 & 37.9 & 118.5 & 13.39 & -5.37 \\
\hline & & & & & 1.013 & 1.547 & 176.2 & 42.2 & 116.1 & 12.24 & -10.91 \\
\hline 4T-abc & S4 & +0.6 & 12.1 & & 1.010 & 1.566 & 175.6 & 60.3 & 117.0 & 12.21 & -4.28 \\
\hline
\end{tabular}




\begin{tabular}{|c|c|c|c|c|c|c|c|c|c|c|c|}
\hline & & & & & 1.011 & 1.543 & 178.2 & 19.9 & 113.7 & 12.33 & -3.27 \\
\hline & & & & & 1.016 & 1.535 & 178.7 & 24.1 & 115.4 & 12.57 & -6.85 \\
\hline \multirow[t]{3}{*}{ 4T-abd } & S4 & +0.9 & 11.8 & & 1.016 & 1.519 & 177.0 & 39.8 & 116.8 & 12.71 & -4.70 \\
\hline & & & & & 1.015 & 1.528 & 173.5 & 83.1 & 119.4 & 12.61 & -9.62 \\
\hline & & & & & 1.016 & 1.525 & 175.5 & 30.9 & 115.5 & 12.63 & -12.47 \\
\hline \multirow{3}{*}{ 4T-acd } & S4 & +2.4 & 11.5 & & 1.016 & 1.513 & 176.0 & 42.2 & 116.6 & 12.79 & -3.93 \\
\hline & & & & & 1.013 & 1.537 & 173.4 & 85.8 & 119.4 & 12.50 & -9.71 \\
\hline & & & & & 1.013 & 1.534 & 174.9 & 13.7 & 113.2 & 12.60 & -7.87 \\
\hline \multirow[t]{3}{*}{ 4T-bed } & S4 & +2.6 & 11.8 & & 1.007 & 1.571 & 174.8 & 22.8 & 115.6 & 11.93 & -12.55 \\
\hline & & & & & 1.011 & 1.540 & 174.1 & 37.4 & 113.6 & 12.28 & -1.86 \\
\hline & & & & & 1.024 & 1.499 & 178.4 & 20.5 & 118.1 & 13.22 & -5.01 \\
\hline \multirow[t]{3}{*}{ 4T-acc } & S4 & +2.0 & 11.8 & & 1.010 & 1.566 & 175.6 & 64.3 & 117.1 & 12.53 & -4.69 \\
\hline & & & & & 1.011 & 1.543 & 178.2 & 27.4 & 113.7 & 12.37 & -2.54 \\
\hline & & & & & 1.016 & 1.535 & 178.7 & 6.0 & 117.0 & 12.46 & -2.37 \\
\hline \multirow[t]{3}{*}{ 4T-ced } & S4 & +2.8 & 12.0 & & 1.007 & 1.582 & 171.6 & 28.4 & 113.7 & 11.85 & -6.13 \\
\hline & & & & & 1.011 & 1.538 & 175.9 & 34.4 & 113.4 & 12.38 & -1.16 \\
\hline & & & & & 1.024 & 1.499 & 177.5 & 17.7 & 117.7 & 13.48 & -4.40 \\
\hline \multirow[t]{3}{*}{ 4T-add } & $\mathrm{S} 4$ & +0.6 & 12.6 & & 1.018 & 1.511 & 179.1 & 47.8 & 119.4 & 13.22 & -11.50 \\
\hline & & & & & 1.015 & 1.529 & 176.8 & 50.4 & 119.3 & 12.83 & -11.60 \\
\hline & & & & & 1.014 & 1.528 & 177.1 & 41.3 & 117.3 & 12.77 & -7.51 \\
\hline \multirow[t]{3}{*}{ 4T-bdd } & $\mathrm{S} 4$ & +0.4 & 12.7 & & 1.014 & 1.525 & 177.8 & 46.2 & 119.6 & 12.91 & -11.24 \\
\hline & & & & & 1.007 & 1.562 & 177.0 & 66.1 & 119.1 & 11.95 & -9.71 \\
\hline & & & & & 1.017 & 1.523 & 177.4 & 30.0 & 115.7 & 12.67 & -10.94 \\
\hline \multirow[t]{3}{*}{ 4T-cdd } & S4 & +2.5 & 12.3 & & 1.007 & 1.582 & 171.6 & 28.4 & 113.7 & 13.19 & -10.96 \\
\hline & & & & & 1.011 & 1.538 & 175.9 & 34.4 & 113.4 & 12.97 & -11.90 \\
\hline & & & & & 1.024 & 1.499 & 177.5 & 17.7 & 117.7 & 12.23 & -6.32 \\
\hline \multirow[t]{3}{*}{ 4T-ddd } & S4 & +1.5 & 12.7 & & 1.013 & 1.530 & 178.3 & 62.5 & 119.5 & 12.75 & -12.68 \\
\hline & & & & & 1.011 & 1.534 & 177.4 & 57.9 & 118.3 & 12.67 & -10.46 \\
\hline & & & & & 1.014 & 1.523 & 177.3 & 57.0 & 118.7 & 12.97 & -12.44 \\
\hline $5 \mathrm{M}-\mathrm{a}$ & $6 a$ & 0 & - & & 0.965 & - & - & 15.1 & 114.9 & 4.01 & -15.19 \\
\hline $5 M-b$ & $6 a$ & +0.5 & - & & 0.965 & - & - & 0.2 & 113.1 & 3.68 & -14.09 \\
\hline 5M-c & $6 a$ & +0.9 & - & & 0.965 & - & - & 7.9 & 113.8 & 4.06 & -21.90 \\
\hline 5M-d & $6 a$ & +1.4 & - & & 0.965 & - & - & 16.5 & 115.5 & 4.53 & -20.50 \\
\hline 5M-e & $6 a$ & +1.6 & - & & 0.964 & - & - & 0.1 & 116.5 & 4.18 & -14.38 \\
\hline \multirow[t]{2}{*}{ 5D-aa } & $6 \mathrm{~b}$ & 0 & 10.6 & 4.7 & 1.010 & 1.573 & 174.9 & 13.2 & 115.8 & 12.34 & -15.04 \\
\hline & & & & & 1.010 & 1.573 & 174.9 & 13.2 & 115.8 & 12.34 & -15.05 \\
\hline \multirow[t]{2}{*}{ 5D-cc } & $6 b$ & +0.6 & 11.1 & & 1.012 & 1.563 & 174.3 & 18.4 & 115.6 & 12.44 & -19.51 \\
\hline & & & & & 1.012 & 1.563 & 174.3 & 18.4 & 115.6 & 12.44 & -19.51 \\
\hline \multirow[t]{2}{*}{ 5D-ce } & $6 b$ & +2.2 & 10.7 & & 1.010 & 1.570 & 174.2 & 2.6 & 117.3 & 12.63 & -17.64 \\
\hline & & & & & 1.009 & 1.579 & 174.5 & 16.5 & 115.1 & 12.44 & -19.28 \\
\hline \multirow[t]{2}{*}{ 5D-ee } & $6 b$ & +3.6 & 10.4 & & 1.007 & 1.586 & 174.2 & 10.7 & 117.2 & 12.48 & -17.18 \\
\hline & & & & & 1.008 & 1.581 & 174.4 & 4.5 & 117.2 & 12.53 & -17.64 \\
\hline \multirow[t]{3}{*}{$5 \mathrm{~T}$} & S5 & 0 & 13.4 & & 1.011 & 1.544 & 177.7 & 29.5 & 116.8 & 12.57 & -19.68 \\
\hline & & & & & 1.011 & 1.552 & 176.4 & 60.1 & 118.1 & 12.76 & -21.43 \\
\hline & & & & & 1.012 & 1.537 & 178.8 & 34.0 & 116.2 & 12.59 & -18.96 \\
\hline
\end{tabular}

a - Averaged energy per one hydrogen bond.

$\mathrm{b}$ - Positive values were taken in all cases except for $\mathrm{PhHPOOH}$ acid, where the negative sign means that $\mathrm{OH}$ proton is on the same side of POO plane as -H substituent is located. 
Table S2. Hydrogen bond energies (BSSE corrected; in kcal/mol per one bond), selected equilibrium geometric and spectral parameters for monomers and cyclic dimers of hypophosphinic acid (1M and 1D-a) and dimethylphosphinic acid (2M and 2D-a), calculated at various levels of theory and various basis sets. The $\mathrm{O}-\mathrm{H}$ bond length $(r(\mathrm{OH}))$ and the distance from bridging proton to $\mathrm{P}=\mathrm{O}$ oxygen $(r(\mathrm{H} \cdots \mathrm{O}))$ are given in $\AA$; the hydrogen bond angle $(\angle \mathrm{OHO})$ and the dihedral angle $(\angle \mathrm{OPOH})-$ in degrees. The ${ }^{1} \mathrm{H}$ and ${ }^{31} \mathrm{P}$ NMR chemical shifts $(\delta \mathrm{H}$ and $\delta \mathrm{P})$ are given in ppm.

\begin{tabular}{|c|c|c|c|c|c|c|c|}
\hline Complex & $\Delta E_{\mathrm{HB}}$ & $r(\mathrm{OH})$ & $r(\mathrm{H} \cdots \mathrm{O})$ & $\angle \mathrm{OHO}$ & $\angle \mathrm{OPOH}$ & $\delta \mathrm{H}$ & $\delta \mathrm{P}$ \\
\hline \multicolumn{8}{|c|}{ Geometry/NMR: B3LYP/6-311++G(d,p) } \\
\hline $1 \mathrm{M}$ & - & 0.965 & - & - & 39.9 & 3.45 & -16.80 \\
\hline \multirow[t]{2}{*}{ 1D-a } & 11.3 & 1.005 & 1.623 & 166.3 & 40.2 & 11.49 & -9.60 \\
\hline & & 1.005 & 1.622 & 166.3 & -40.3 & 11.49 & -9.60 \\
\hline \multicolumn{8}{|c|}{ Geometry/NMR: MP2/aug-cc-pVDZ } \\
\hline $1 \mathrm{M}$ & - & 0.972 & - & - & 31.9 & 2.42 & -22.71 \\
\hline \multirow[t]{2}{*}{ 1D-a } & 9.7 & 1.011 & 1.610 & 170.1 & 46.2 & 10.38 & -13.35 \\
\hline & & 1.011 & 1.607 & 170.2 & 46.2 & 10.42 & -13.36 \\
\hline \multicolumn{8}{|c|}{ Geometry: MP2/aug-cc-pVDZ; NMR: MP2/ pcS-2 } \\
\hline $1 \mathrm{M}$ & - & - & - & - & - & 2.9 & -9.78 \\
\hline \multirow[t]{2}{*}{ 1D-a } & - & - & - & - & - & 10.97 & 0.18 \\
\hline & - & - & - & - & - & 11.01 & 0.17 \\
\hline
\end{tabular}


Table S3. Calculated (B3LYP/6-311++(d,p)) distances from phosphorous atom to its immediate neighbors of monomers, cyclic dimers and cyclic trimers of phosphinic and phosphoric acids $\mathbf{1}-\mathbf{5}$. The $\mathrm{P}-\mathrm{OH}, \mathrm{P}=\mathrm{O}$, $\mathrm{P}-\mathrm{H}, \mathrm{P}-\mathrm{C}$ and P-OC distances are given in $\AA$.

\begin{tabular}{|c|c|c|c|c|c|c|c|c|c|}
\hline Complex & Figure & $\begin{array}{c}r(\mathrm{P}-\mathrm{OH}), \\
\AA\end{array}$ & $\begin{array}{c}r(\mathrm{P}=\mathrm{O}), \\
\AA\end{array}$ & $\begin{array}{c}r(\mathrm{P}-\mathrm{H}), \\
\AA\end{array}$ & $\begin{array}{c}r(\mathrm{P}-\mathrm{H}), \\
\AA\end{array}$ & $\begin{array}{c}r(\mathrm{P}-\mathrm{C}), \\
\AA\end{array}$ & $\begin{array}{c}r(\mathrm{P}-\mathrm{C}), \\
\AA\end{array}$ & $\begin{array}{c}r(\mathrm{P}-\mathrm{OC}) \\
\AA\end{array}$ & $\begin{array}{c}r(\mathrm{P}-\mathrm{OC}), \\
\AA\end{array}$ \\
\hline $1 \mathrm{M}$ & $2 a$ & 1.628 & 1.483 & 1.415 & 1.409 & - & - & - & - \\
\hline \multirow[t]{2}{*}{ 1D-a } & $2 b$ & 1.588 & 1.505 & 1.413 & 1.406 & - & - & - & - \\
\hline & & 1.588 & 1.505 & 1.406 & 1.413 & - & - & - & - \\
\hline \multirow[t]{2}{*}{ 1D-b } & $2 b$ & 1.588 & 1.504 & 1.413 & 1.407 & - & - & - & - \\
\hline & & 1.588 & 1.504 & 1.413 & 1.407 & - & - & - & - \\
\hline \multirow[t]{3}{*}{ 1T-a } & $2 c$ & 1.578 & 1.505 & 1.405 & 1.413 & - & - & - & - \\
\hline & & 1.578 & 1.505 & 1.406 & 1.411 & - & - & - & - \\
\hline & & 1.578 & 1.505 & 1.413 & 1.405 & - & - & - & - \\
\hline \multirow[t]{3}{*}{ 1T-b } & $2 c$ & 1.579 & 1.505 & 1.411 & 1.406 & - & - & - & - \\
\hline & & 1.579 & 1.505 & 1.411 & 1.406 & - & - & - & - \\
\hline & & 1.579 & 1.505 & 1.411 & 1.406 & - & - & - & - \\
\hline $2 M$ & S1a & 1.645 & 1.491 & - & - & 1.816 & 1.816 & - & - \\
\hline \multirow{2}{*}{ 2D-a } & S1b & 1.598 & 1.513 & - & - & 1.818 & 1.810 & - & - \\
\hline & & 1.598 & 1.513 & - & - & 1.810 & $\begin{array}{l}1.818 \\
\end{array}$ & - & - \\
\hline \multirow{2}{*}{ 2D-b } & S1b & 1.596 & 1.513 & - & - & 1.817 & 1.812 & - & - \\
\hline & & 1.596 & 1.513 & - & - & 1.817 & 1.812 & - & - \\
\hline \multirow[t]{3}{*}{$2 \mathrm{~T}-\mathrm{a}$} & S1c & 1.590 & 1.514 & - & - & 1.818 & 1.811 & - & - \\
\hline & & 1.591 & 1.514 & - & - & 1.812 & 1.816 & - & - \\
\hline & & 1.591 & 1.514 & - & - & 1.811 & 1.818 & - & - \\
\hline \multirow[t]{3}{*}{ 2T-b } & S1c & 1.589 & 1.514 & - & - & 1.817 & 1.812 & - & - \\
\hline & & 1.590 & 1.514 & - & - & 1.812 & 1.817 & - & - \\
\hline & & 1.590 & 1.514 & - & - & 1.817 & 1.812 & - & - \\
\hline $3 M$ & $3 a$ & 1.636 & 1.487 & 1.416 & - & 1.806 & - & - & - \\
\hline \multirow{2}{*}{ 3D-a } & $3 b$ & 1.592 & 1.509 & 1.408 & - & 1.812 & - & - & - \\
\hline & & 1.598 & 1.509 & 1.408 & - & 1.812 & - & - & - \\
\hline \multirow{2}{*}{ 3D-b } & $3 b$ & 1.592 & 1.509 & 1.414 & - & 1.804 & - & - & - \\
\hline & & 1.592 & 1.509 & 1.408 & - & 1.812 & - & - & - \\
\hline \multirow{2}{*}{ 3D-c } & $3 b$ & 1.592 & 1.509 & 1.414 & - & 1.804 & - & - & - \\
\hline & & 1.592 & 1.509 & 1.414 & - & 1.804 & - & - & - \\
\hline \multirow{2}{*}{ 3D-d } & S2a & 1.592 & 1.508 & 1.414 & - & 1.804 & - & - & - \\
\hline & & 1.591 & 1.509 & 1.409 & - & 1.812 & - & - & - \\
\hline \multirow[t]{2}{*}{ 3D-e } & $\mathrm{S} 2 \mathrm{a}$ & 1.592 & 1.508 & 1.409 & - & 1.812 & - & - & - \\
\hline & & 1.592 & 1.508 & 1.409 & - & 1.812 & - & - & - \\
\hline \multirow[t]{2}{*}{ 3D-f } & $\mathrm{S} 2 \mathrm{a}$ & 1.591 & 1.509 & 1.414 & - & 1.805 & - & - & - \\
\hline & & 1.591 & 1.509 & 1.414 & - & 1.805 & - & - & - \\
\hline \multirow[t]{3}{*}{ 3T-a } & 4 & 1.583 & 1.509 & 1.412 & - & 1.807 & - & - & - \\
\hline & & 1.583 & 1.509 & 1.412 & - & 1.807 & - & - & - \\
\hline & & 1.583 & 1.509 & 1.412 & - & 1.807 & - & - & - \\
\hline \multirow[t]{3}{*}{ 3T-b } & 4 & 1.582 & 1.510 & 1.408 & - & 1.812 & - & - & - \\
\hline & & 1.583 & 1.509 & 1.412 & - & 1.807 & - & - & - \\
\hline & & 1.584 & 1.508 & 1.412 & - & 1.807 & - & - & - \\
\hline \multirow[t]{3}{*}{ 3T-c } & 4 & 1.584 & 1.507 & 1.413 & - & 1.807 & - & - & - \\
\hline & & 1.582 & 1.509 & 1.408 & - & 1.813 & - & - & - \\
\hline & & 1.581 & 1.509 & 1.408 & - & 1.813 & - & - & - \\
\hline \multirow[t]{3}{*}{ 3T-d } & 4 & 1.585 & 1.507 & 1.408 & - & 1.814 & - & - & - \\
\hline & & 1.583 & 1.508 & 1.408 & - & 1.814 & - & - & - \\
\hline & & 1.582 & 1.509 & 1.408 & - & 1.814 & - & - & - \\
\hline 3T-e & $\mathrm{S} 2 \mathrm{~b}$ & 1.583 & 1.509 & 1.412 & - & 1.807 & - & - & - \\
\hline & & 1.583 & 1.509 & 1.413 & - & 1.805 & - & - & - \\
\hline & & 1.583 & 1.509 & 1.413 & - & 1.806 & - & - & - \\
\hline 3T-f & $\mathrm{S} 2 \mathrm{~b}$ & 1.581 & 1.510 & 1.408 & - & 1.812 & - & - & - \\
\hline
\end{tabular}




\begin{tabular}{|c|c|c|c|c|c|c|c|c|c|}
\hline & & 1.583 & 1.509 & 1.414 & - & 1.805 & - & - & - \\
\hline & & 1.583 & 1.509 & 1.413 & - & 1.806 & - & - & - \\
\hline \multirow[t]{3}{*}{ 3T-g } & S2b & 1.584 & 1.508 & 1.412 & - & 1.807 & - & - & - \\
\hline & & 1.582 & 1.508 & 1.407 & - & 1.814 & - & - & - \\
\hline & & 1.583 & 1.509 & 1.413 & - & 1.805 & - & - & - \\
\hline \multirow[t]{3}{*}{ 3T-h } & $\mathrm{S} 2 \mathrm{~b}$ & 1.583 & 1.509 & 1.412 & - & 1.807 & - & - & - \\
\hline & & 1.583 & 1.509 & 1.414 & - & 1.806 & - & - & - \\
\hline & & 1.582 & 1.509 & 1.407 & - & 1.813 & - & - & - \\
\hline \multirow[t]{3}{*}{$3 T-i$} & $\mathrm{~S} 2 \mathrm{~b}$ & 1.582 & 1.509 & 1.408 & - & 1.812 & - & - & - \\
\hline & & 1.582 & 1.508 & 1.407 & - & 1.815 & - & - & - \\
\hline & & 1.584 & 1.508 & 1.413 & - & 1.806 & - & - & - \\
\hline \multirow[t]{3}{*}{$3 \mathrm{~T}-\mathrm{j}$} & $\mathrm{S} 2 \mathrm{~b}$ & 1.582 & 1.510 & 1.408 & - & 1.812 & - & - & - \\
\hline & & 1.583 & 1.508 & 1.414 & - & 1.806 & - & - & - \\
\hline & & 1.582 & 1.508 & 1.407 & - & 1.814 & - & - & - \\
\hline \multirow[t]{3}{*}{ 3T-k } & S2b & 1.584 & 1.508 & 1.412 & - & 1.807 & - & - & - \\
\hline & & 1.583 & 1.508 & 1.407 & - & 1.814 & - & - & - \\
\hline & & 1.583 & 1.508 & 1.407 & - & 1.813 & - & - & - \\
\hline \multirow{3}{*}{ 3T-I } & $\mathrm{S} 2 \mathrm{~b}$ & 1.583 & 1.509 & 1.408 & - & 1.812 & - & - & - \\
\hline & & 1.583 & 1.507 & 1.407 & - & 1.815 & - & - & - \\
\hline & & 1.584 & 1.507 & 1.407 & - & 1.814 & - & - & - \\
\hline 4M-a & $5 a$ & 1.612 & 1.479 & - & - & - & - & 1.601 & 1.596 \\
\hline 4M-b & $5 a$ & 1.629 & 1.472 & - & - & - & - & 1.602 & 1.591 \\
\hline $4 M-c$ & $5 a$ & 1.628 & 1.480 & - & - & - & - & 1.593 & 1.593 \\
\hline 4M-d & $5 a$ & 1.613 & 1.472 & - & - & - & - & 1.615 & 1.595 \\
\hline \multirow{2}{*}{ 4D-aa } & S3 & 1.570 & 1.500 & - & - & - & - & 1.600 & 1.593 \\
\hline & & 1.570 & 1.500 & - & - & - & - & 1.593 & 1.600 \\
\hline \multirow[t]{2}{*}{ 4D-ab } & $5 b$ & 1.584 & 1.491 & - & - & - & - & 1.600 & 1.591 \\
\hline & & 1.569 & 1.500 & - & - & - & - & 1.593 & 1.600 \\
\hline \multirow[t]{2}{*}{ 4D-ac } & S3 & 1.582 & 1.499 & - & - & - & - & 1.593 & 1.591 \\
\hline & & 1.569 & 1.500 & - & - & - & - & 1.594 & 1.600 \\
\hline \multirow[t]{2}{*}{ 4D-ad } & S3 & 1.570 & 1.492 & - & - & - & - & 1.599 & 1.608 \\
\hline & & 1.570 & 1.500 & - & - & - & - & 1.593 & 1.599 \\
\hline \multirow{2}{*}{ 4D-bb } & $5 b$ & 1.583 & 1.492 & - & - & - & - & 1.600 & 1.591 \\
\hline & & 1.583 & 1.492 & - & - & - & - & 1.591 & 1.600 \\
\hline \multirow{2}{*}{ 4D-bc } & $5 b$ & 1.584 & 1.491 & - & - & - & - & 1.599 & 1.592 \\
\hline & & 1.582 & 1.500 & - & - & - & - & 1.593 & 1.591 \\
\hline \multirow[t]{2}{*}{ 4D-bd } & $5 b$ & 1.584 & 1.492 & - & - & - & - & 1.599 & 1.591 \\
\hline & & 1.569 & 1.493 & - & - & - & - & 1.607 & 1.599 \\
\hline \multirow[t]{2}{*}{ 4D-cc } & S3 & 1.582 & 1.499 & - & - & - & - & 1.594 & 1.591 \\
\hline & & 1.582 & 1.499 & - & - & - & - & 1.594 & 1.591 \\
\hline \multirow[t]{2}{*}{ 4D-cd } & S3 & 1.583 & 1.500 & - & - & - & - & 1.593 & 1.590 \\
\hline & & 1.570 & 1.492 & - & - & - & - & 1.608 & 1.599 \\
\hline \multirow[t]{2}{*}{ 4D-dd } & S3 & 1.570 & 1.493 & - & - & - & - & 1.598 & 1.607 \\
\hline & & 1.570 & 1.493 & - & - & - & - & 1.607 & 1.598 \\
\hline 4T-aaa & S4 & 1.563 & 1.496 & - & - & - & - & 1.598 & 1.602 \\
\hline & & 1.564 & 1.497 & - & - & - & - & 1.595 & 1.602 \\
\hline & & 1.565 & 1.497 & - & - & - & - & 1.595 & 1.602 \\
\hline 4T-aab & S4 & 1.562 & 1.497 & - & - & - & - & 1.597 & 1.602 \\
\hline & & 1.576 & 1.490 & - & - & - & - & 1.593 & 1.602 \\
\hline & & 1.563 & 1.498 & - & - & - & - & 1.595 & 1.602 \\
\hline 4T-aac & S4 & 1.569 & 1.496 & - & - & - & - & 1.595 & 1.599 \\
\hline & & 1.577 & 1.500 & - & - & - & - & 1.594 & 1.592 \\
\hline & & 1.563 & 1.504 & - & - & - & - & 1.592 & 1.601 \\
\hline 4T-aad & S4 & 1.564 & 1.497 & - & - & - & - & 1.597 & 1.600 \\
\hline & & 1.565 & 1.488 & - & - & - & - & 1.610 & 1.599 \\
\hline & & 1.565 & 1.498 & - & - & - & - & 1.593 & 1.602 \\
\hline 4T-bba & S4 & 1.578 & 1.490 & - & - & - & - & 1.608 & 1.586 \\
\hline
\end{tabular}




\begin{tabular}{|c|c|c|c|c|c|c|c|c|c|}
\hline & & 1.560 & 1.501 & - & - & - & - & 1.606 & 1.593 \\
\hline & & 1.578 & 1.489 & - & - & - & - & 1.603 & 1.593 \\
\hline \multirow[t]{3}{*}{ 4T-bbb } & S4 & 1.577 & 1.491 & - & - & - & - & 1.608 & 1.587 \\
\hline & & 1.572 & 1.494 & - & - & - & - & 1.596 & 1.601 \\
\hline & & 1.578 & 1.491 & - & - & - & - & 1.601 & 1.592 \\
\hline \multirow[t]{3}{*}{ 4T-bbc } & S4 & 1.579 & 1.491 & - & - & - & - & 1.607 & 1.586 \\
\hline & & 1.575 & 1.502 & - & - & - & - & 1.596 & 1.592 \\
\hline & & 1.577 & 1.489 & - & - & - & - & 1.600 & 1.597 \\
\hline \multirow[t]{3}{*}{ 4T-bbd } & S4 & 1.576 & 1.493 & - & - & - & - & 1.607 & 1.586 \\
\hline & & 1.560 & 1.495 & - & - & - & - & 1.610 & 1.602 \\
\hline & & 1.577 & 1.491 & - & - & - & - & 1.601 & 1.592 \\
\hline \multirow[t]{3}{*}{ 4T-abc } & S4 & 1.571 & 1.496 & - & - & - & - & 1.596 & 1.598 \\
\hline & & 1.576 & 1.502 & - & - & - & - & 1.590 & 1.595 \\
\hline & & 1.577 & 1.495 & - & - & - & - & 1.600 & 1.592 \\
\hline \multirow[t]{3}{*}{ 4T-abd } & S4 & 1.561 & 1.501 & - & - & - & - & 1.594 & 1.601 \\
\hline & & 1.565 & 1.491 & - & - & - & - & 1.607 & 1.603 \\
\hline & & 1.573 & 1.490 & - & - & - & - & 1.602 & 1.594 \\
\hline \multirow[t]{3}{*}{ 4T-acd } & S4 & 1.563 & 1.501 & - & - & - & - & 1.594 & 1.601 \\
\hline & & 1.566 & 1.490 & - & - & - & - & 1.609 & 1.603 \\
\hline & & 1.573 & 1.497 & - & - & - & - & 1.593 & 1.596 \\
\hline \multirow[t]{3}{*}{ 4T-bed } & S4 & 1.581 & 1.488 & - & - & - & - & 1.605 & 1.588 \\
\hline & & 1.574 & 1.505 & - & - & - & - & 1.591 & 1.592 \\
\hline & & 1.564 & 1.494 & - & - & - & - & 1.605 & 1.605 \\
\hline \multirow[t]{3}{*}{ 4T-acc } & S4 & 1.569 & 1.497 & - & - & - & - & 1.598 & 1.598 \\
\hline & & 1.576 & 1.501 & - & - & - & - & 1.594 & 1.593 \\
\hline & & 1.577 & 1.503 & - & - & - & - & 1.589 & 1.594 \\
\hline \multirow[t]{3}{*}{ 4T-ced } & S4 & 1.54 & 1.496 & - & - & - & - & 1.593 & 1.591 \\
\hline & & 1.574 & 1.505 & - & - & - & - & 1.591 & 1.591 \\
\hline & & 1.563 & 1.495 & - & - & - & - & 1.610 & 1.600 \\
\hline \multirow[t]{3}{*}{ 4T-add } & S4 & 1.562 & 1.491 & - & - & - & - & 1.609 & 1.600 \\
\hline & & 1.564 & 1.491 & - & - & - & - & 1.608 & 1.598 \\
\hline & & 1.562 & 1.499 & - & - & - & - & 1.594 & 1.601 \\
\hline \multirow[t]{3}{*}{ 4T-bdd } & S4 & 1.564 & 1.489 & - & - & - & - & 1.615 & 1.598 \\
\hline & & 1.569 & 1.491 & - & - & - & - & 1.607 & 1.597 \\
\hline & & 1.575 & 1.492 & - & - & - & - & 1.601 & 1.592 \\
\hline \multirow[t]{3}{*}{ 4T-cdd } & S4 & 1.584 & 1.496 & - & - & - & - & 1.593 & 1.591 \\
\hline & & 1.574 & 1.505 & - & - & - & - & 1.591 & 1.591 \\
\hline & & 1.563 & 1.495 & - & - & - & - & 1.610 & 1.600 \\
\hline \multirow[t]{3}{*}{ 4T-ddd } & S4 & 1.564 & 1.488 & - & - & - & - & 1.616 & 1.597 \\
\hline & & 1.566 & 1.491 & - & - & - & - & 1.607 & 1.598 \\
\hline & & 1.566 & 1.489 & - & - & - & - & 1.609 & 1.598 \\
\hline 5M-a & $6 a$ & 1.606 & $\begin{array}{l}1.477 \\
\end{array}$ & - & - & - & - & 1.607 & 1.603 \\
\hline 5M-b & $6 a$ & 1.616 & 1.477 & - & - & - & - & 1.602 & 1.602 \\
\hline 5M-c & $6 a$ & 1.620 & 1.471 & - & - & - & - & 1.608 & 1.598 \\
\hline 5M-d & $6 a$ & 1.610 & 1.471 & - & - & - & - & 1.613 & 1.604 \\
\hline 5M-e & $6 a$ & 1.598 & 1.477 & - & - & - & - & 1.610 & 1.610 \\
\hline \multirow[t]{2}{*}{ 5D-aa } & $6 b$ & 1.566 & 1.496 & - & - & - & - & 1.601 & 1.606 \\
\hline & & 1.566 & 1.496 & - & - & - & - & 1.606 & 1.601 \\
\hline \multirow[t]{2}{*}{ 5D-cc } & $6 \mathrm{~b}$ & 1.575 & 1.490 & - & - & - & - & 1.600 & 1.605 \\
\hline & & 1.575 & 1.490 & - & - & - & - & 1.605 & 1.600 \\
\hline \multirow[t]{2}{*}{ 5D-ce } & $6 \mathrm{~b}$ & 1.559 & 1.497 & - & - & - & - & 1.609 & 1.607 \\
\hline & & 1.578 & 1.489 & - & - & - & - & 1.603 & 1.599 \\
\hline 5D-ee & $6 \mathrm{~b}$ & 1.561 & 1.496 & - & - & - & - & 1.605 & 1.609 \\
\hline & & 1.561 & 1.496 & - & - & - & - & 1.606 & 1.608 \\
\hline $5 T$ & S5 & 1.561 & 1.493 & - & - & - & - & 1.602 & 1.610 \\
\hline & & 1.562 & 1.490 & - & - & - & - & 1.609 & 1.607 \\
\hline & & 1.560 & 1.494 & - & - & - & - & 1.601 & 1.611 \\
\hline
\end{tabular}


a)
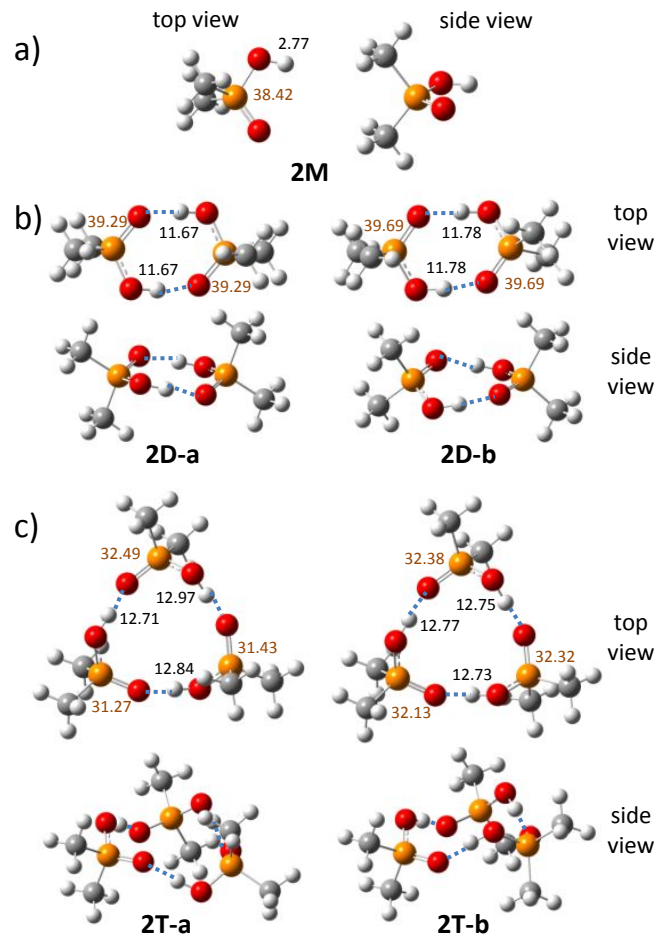

Figure S1. Calculated equilibrium structures (B3LYP/6-311++G(d,p)) of monomer (a), cyclic dimer (b) and cyclic trimer (c) of dimethylphosphinic acid $\mathbf{2}$ in vacuum. All structures are presented in two projections (top and side views). The ${ }^{1} \mathrm{H}$ (black) and ${ }^{31} \mathrm{P}$ (orange) NMR chemical shifts are given in ppm next to corresponding atoms (with $\left(\mathrm{CH}_{3}\right)_{4} \mathrm{Si}$ and $\mathrm{H}_{3} \mathrm{PO}_{4}$ as the reference, respectively). 
a)

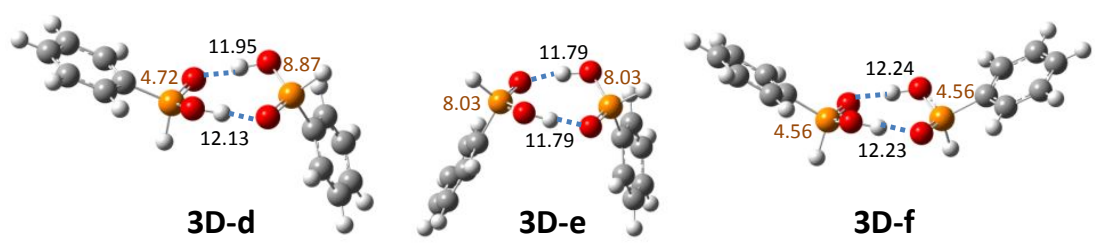

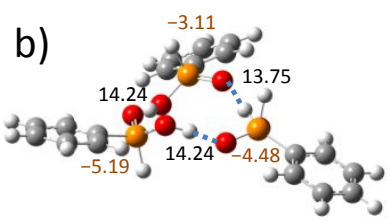

3T-e

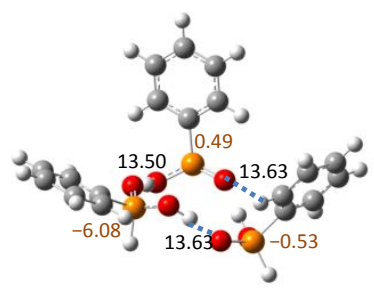

3T-i

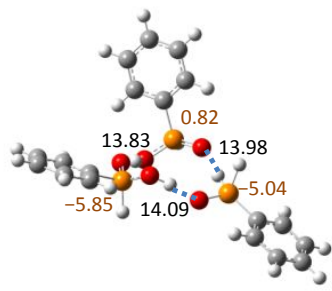

3T-f

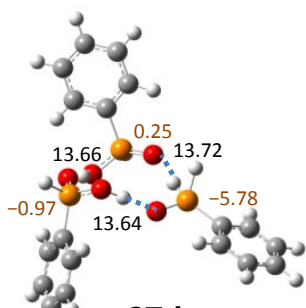

3T-j

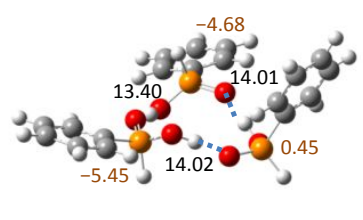

3T-g

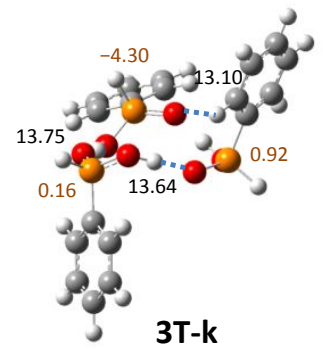

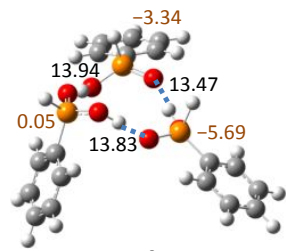

3T-h

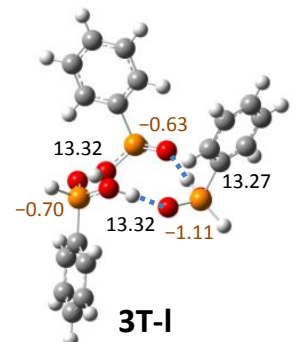

Figure S2. Calculated equilibrium structures (B3LYP/6-311++G(d,p)) of dimer (a) and cyclic trimer (b) of phenylphosphinic acid 3 in vacuum. All structures are presented in side view. The ${ }^{1} \mathrm{H}$ (black) and ${ }^{31} \mathrm{P}$ (orange) NMR chemical shifts are given in ppm next to corresponding atoms (with $\left(\mathrm{CH}_{3}\right)_{4} \mathrm{Si}$ and $\mathrm{H}_{3} \mathrm{PO}_{4}$ as the reference, respectively). 


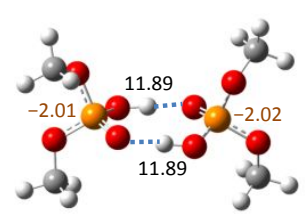

4D-aa

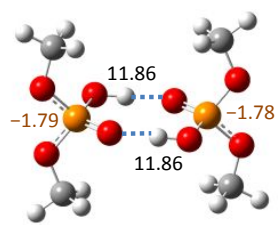

4D-cc

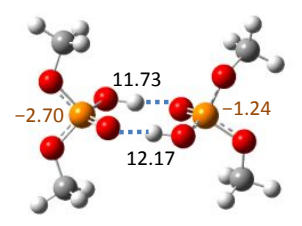

4D-ac

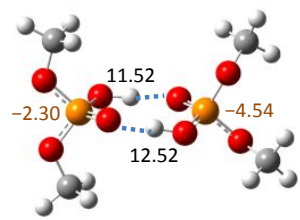

4D-cd

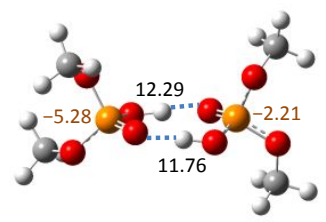

4M-ad

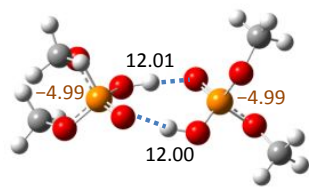

4D-dd

Figure S3. Calculated equilibrium structures (B3LYP/6-311++G(d,p)) of cyclic dimer of dimethylphosphoric acid $\mathbf{4}$ in vacuum. All structures are presented in side view. NMR chemical shifts are given in ppm next to corresponding atoms (with $\left(\mathrm{CH}_{3}\right)_{4} \mathrm{Si}$ and $\mathrm{H}_{3} \mathrm{PO}_{4}$ as the reference, respectively). 


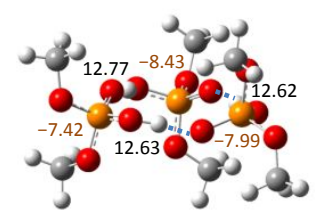

4T-aaa

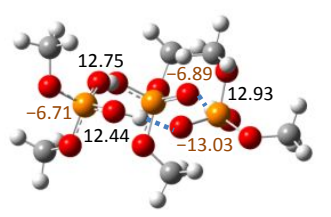

4T-aad

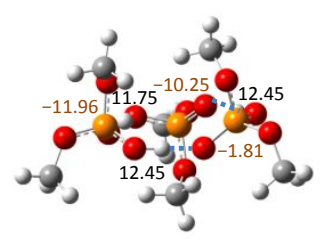

4T-bbc

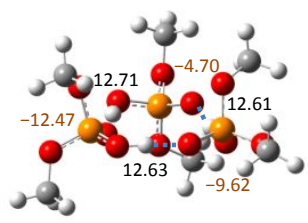

4T-abd
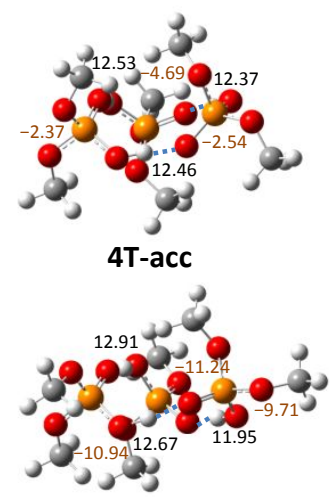

4T-bdd

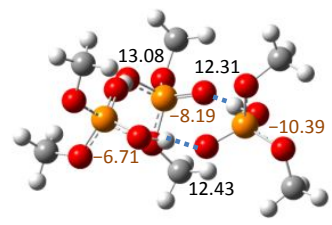

4T-aab
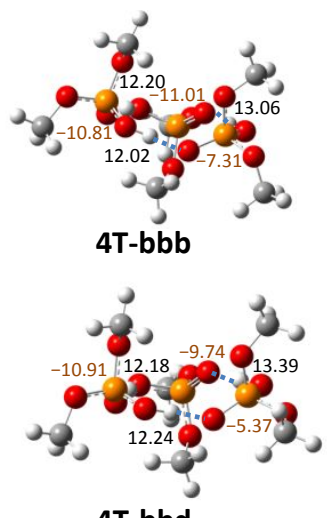

4T-bbd

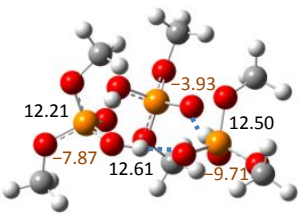

4T-acb

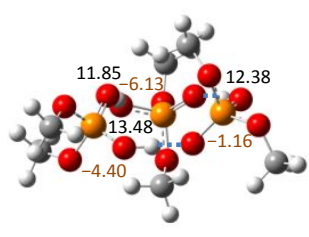

4T-ccd

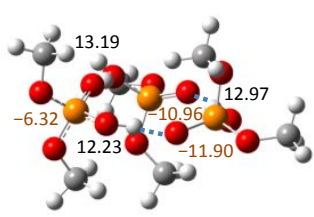

4T-cdd

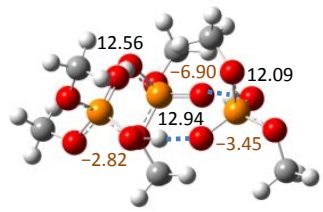

4T-aаc

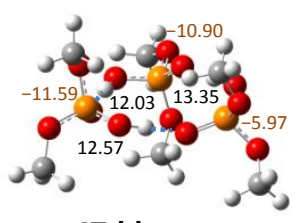

4T-bba

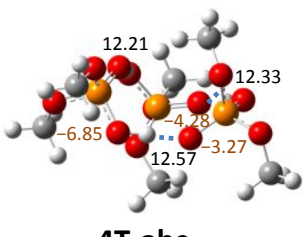

4T-abc

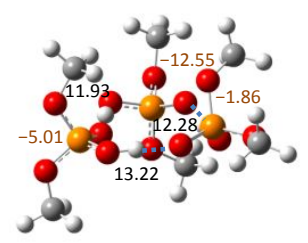

4T-bcd

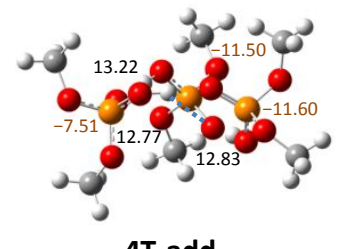

4T-add

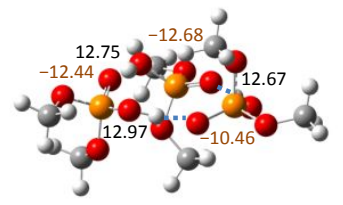

4T-ddd

Figure S4. Calculated equilibrium structures (B3LYP/6-311++G(d,p)) of cyclic trimer of dimethylphosphoric acid 4 in vacuum. All structures are presented in side view. NMR chemical shifts are given in ppm next to corresponding atoms (with $\left(\mathrm{CH}_{3}\right)_{4} \mathrm{Si}$ and $\mathrm{H}_{3} \mathrm{PO}_{4}$ as the reference, respectively). 


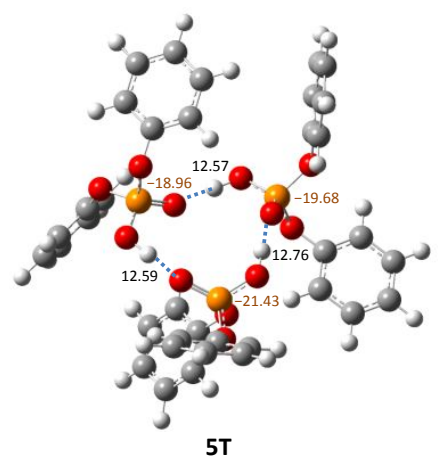

Figure S5. Calculated equilibrium structure (B3LYP/6-311++G(d,p)) of cyclic trimer of diphenylphosphoric acid $\mathbf{5}$ in vacuum. The structure is presented in top view. NMR chemical shifts are given in ppm next to corresponding atoms (with $\left(\mathrm{CH}_{3}\right)_{4} \mathrm{Si}$ and $\mathrm{H}_{3} \mathrm{PO}_{4}$ as the reference, respectively). 


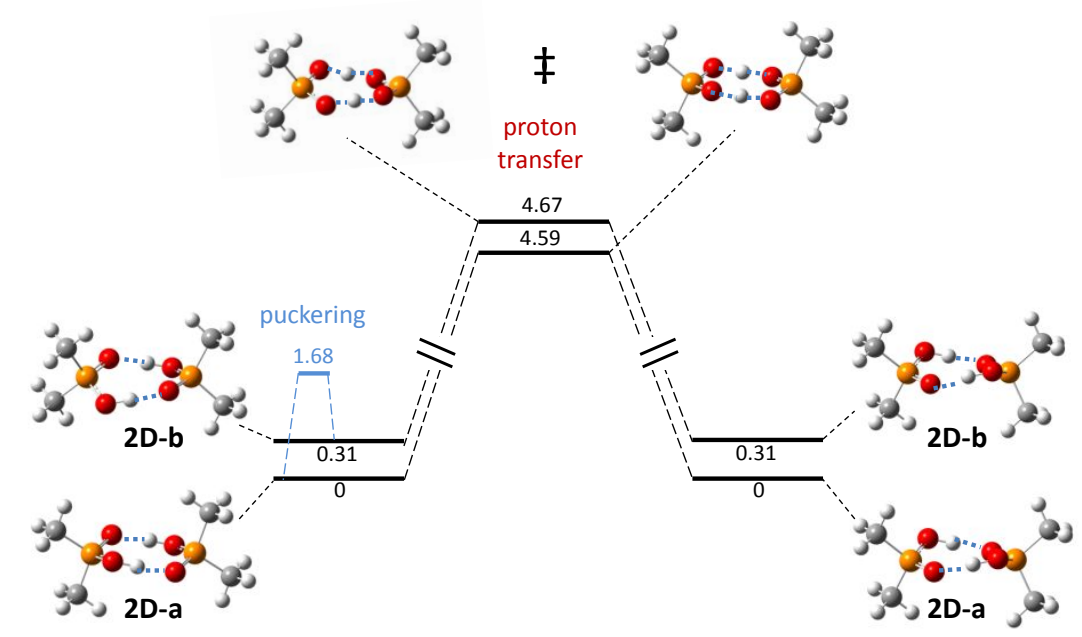

Figure S6. Molecular structures and energy barriers for the proton transfer (PT) and hydrogen bond ring puckering ("twist") of cyclic dimer of dimethylphosphinic acid 2. In red color barrier of PT are given, in blue - energy barrier of puckering. Energies are given in $\mathrm{kcal} / \mathrm{mol}$. 


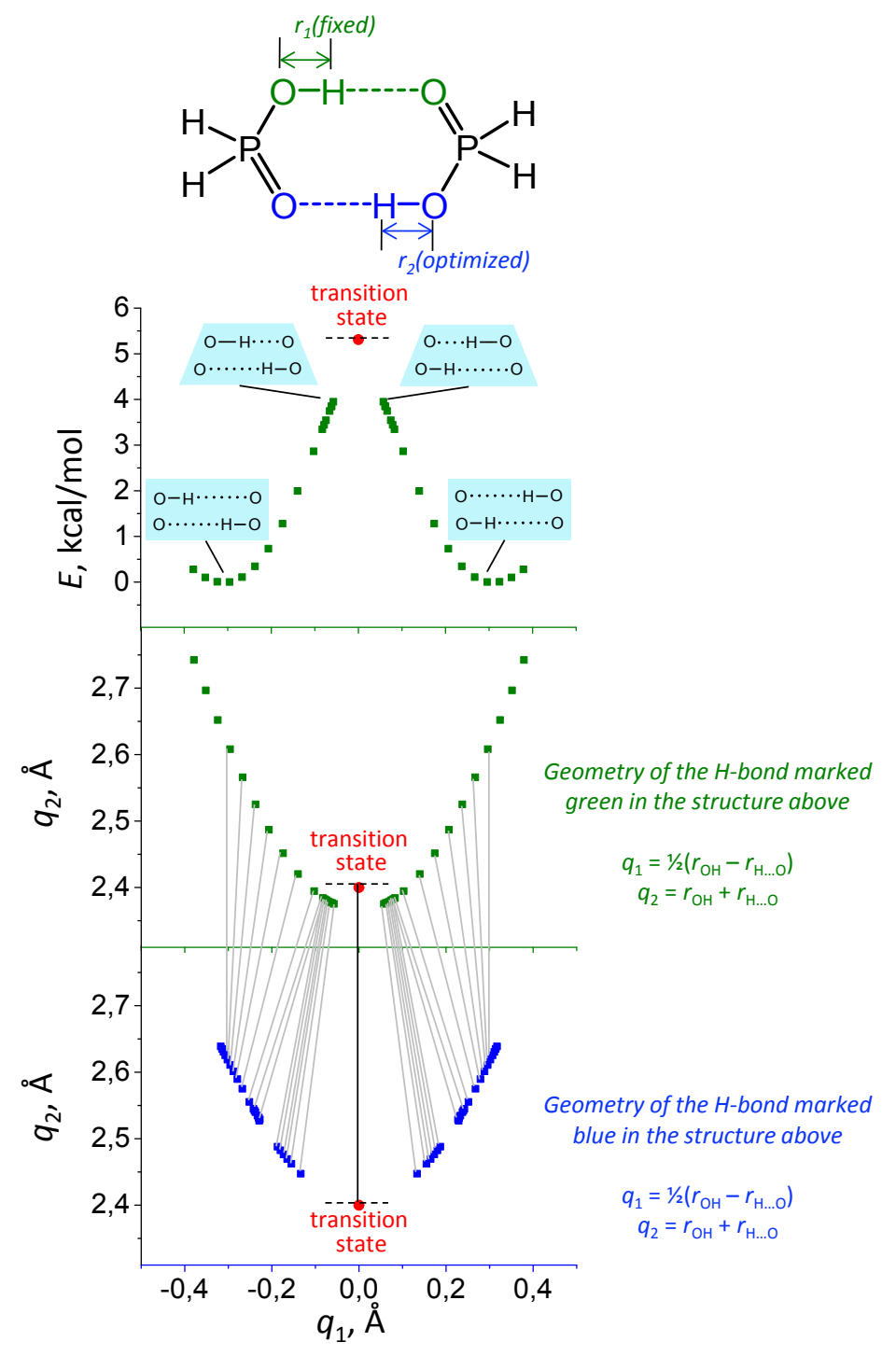

Figure S7. Calculated (B3LYP/6-311++G(d,p)) energy and geometry changes along the proton transfer pathway a cyclic dimer of $\mathrm{H}_{2} \mathrm{POOH}$ acid (1). The $\mathrm{OH}$ distance $r_{1}$ (see the chemical structure above the plots) was changed in a step-wise fashion, while all other geometric parameters were optimized. (top) Potential energy profile along the proton transfer pathway. (middle) Geometry changes of the OHO hydrogen bond marked in green. (bottom) Geometry changes of the OHO hydrogen bond marked in blue.

Comment: the proton is being forced to transfer in the "green" hydrogen bond ( $r_{1}$ changes). At the same time, the geometry of the "blue" hydrogen bond (including $r_{2}$ distance) responds to that and in the transition states both bridging protons are in the centers of their respective hydrogen bonds. However, in most of the other points along the PT pathway the "blue" protons drags behind the "green" one. 


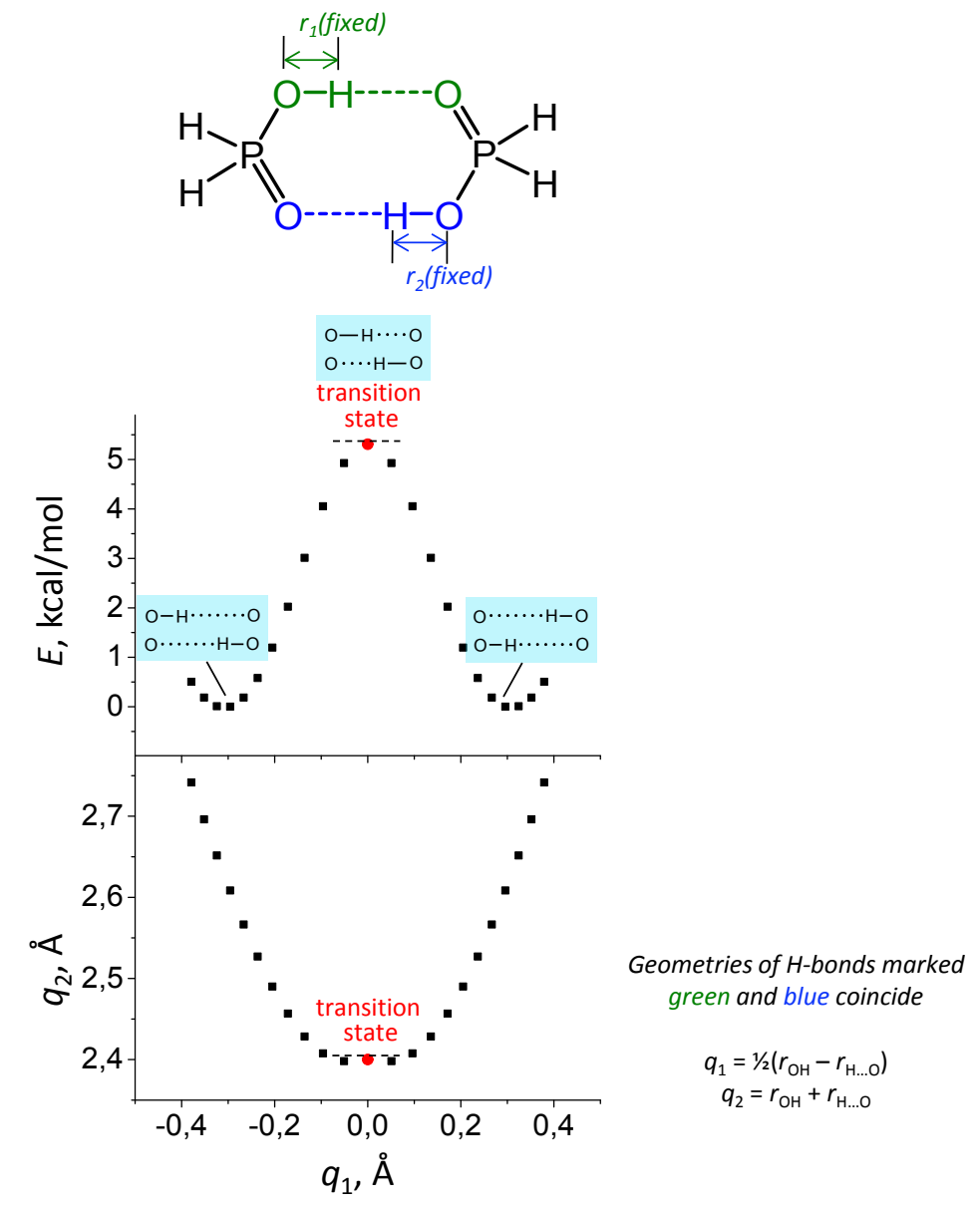

Figure S8. Calculated (B3LYP/6-311++G(d,p)) energy and geometry changes along the proton transfer pathway a cyclic dimer of $\mathrm{H}_{2} \mathrm{POOH}$ acid (1). The $\mathrm{OH}$ distances $r_{1}$ and $r_{2}$ (see the chemical structure above the plots) were kept equal and changed simultaneously in a step-wise fashion, while all other geometric parameters were optimized. (top) Potential energy profile along the proton transfer pathway. (bottom) Geometry changes of the OHO hydrogen bonds (both H-bonds are equal due to the forced symmetry of the system). 


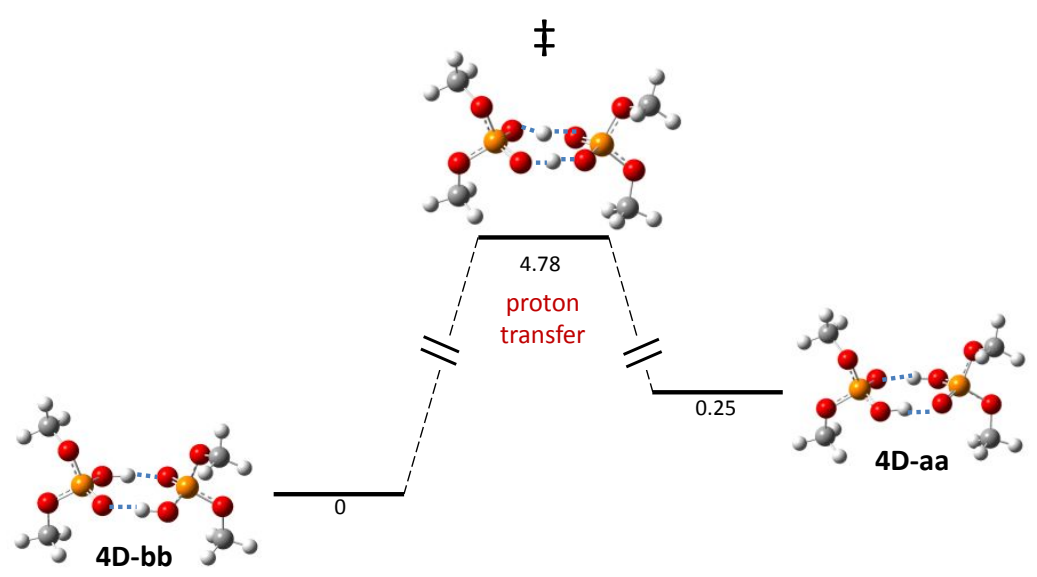

Figure S9. Molecular structures and energy barrier for the proton transfer (PT) in cyclic dimer of dimethylphosphoric acid 4. Energies are given in $\mathrm{kcal} / \mathrm{mol}$. 


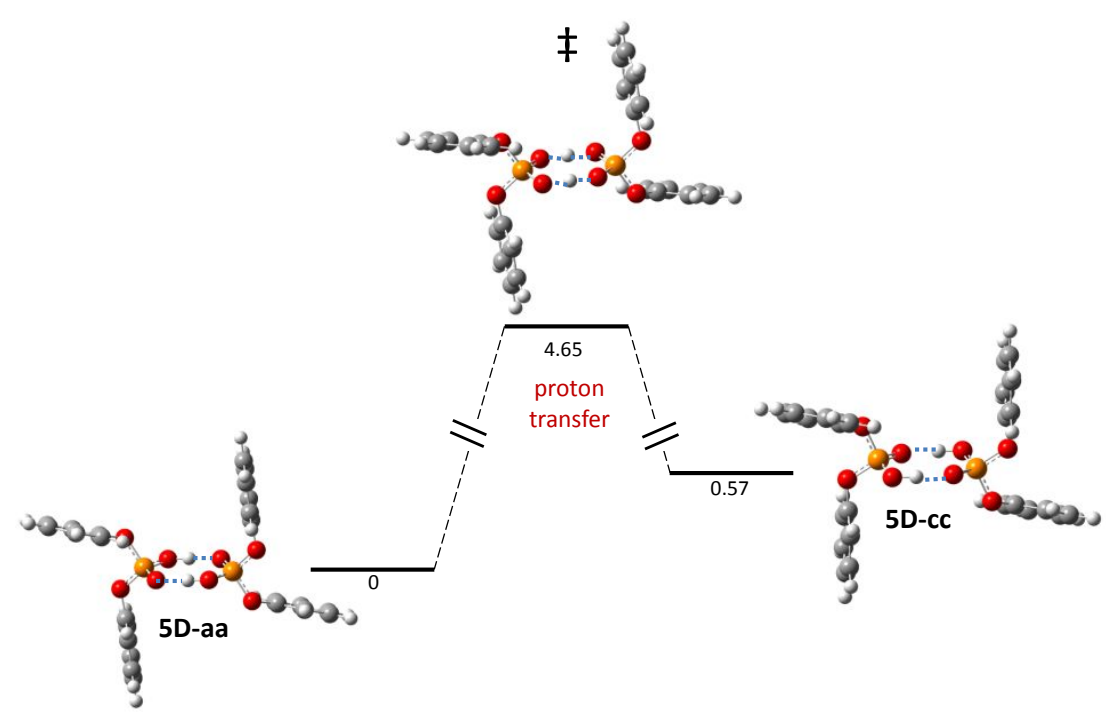

Figure S10. Molecular structures and energy barrier for the proton transfer (PT) in cyclic dimer of diphenylphosphoric acid 5. Energies are given in $\mathrm{kcal} / \mathrm{mol}$. 


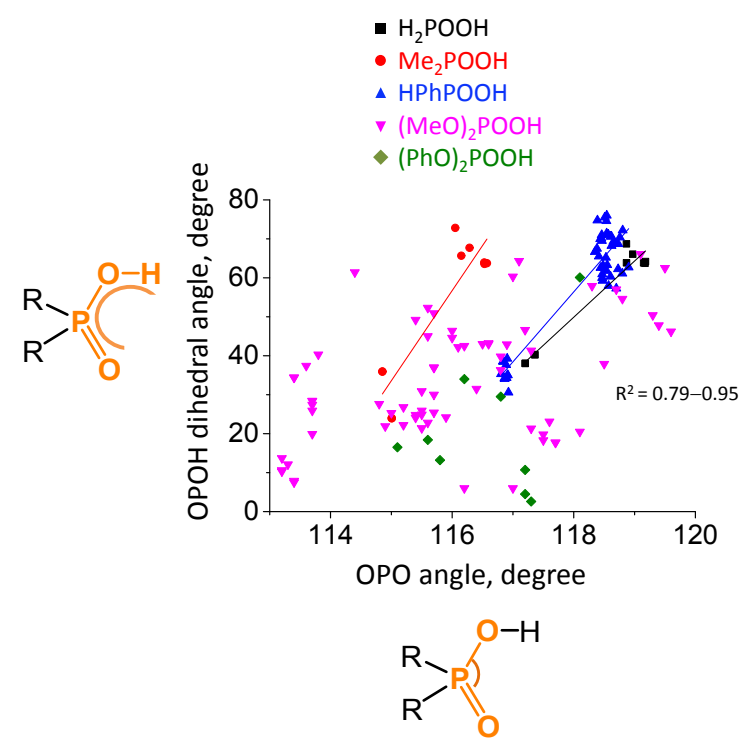

Figure S11. The interdependence of the $\mathrm{OPO}$ angle and $\mathrm{OPOH}$ dihedral angle for cyclic dimers and trimers of acids 1-5. 


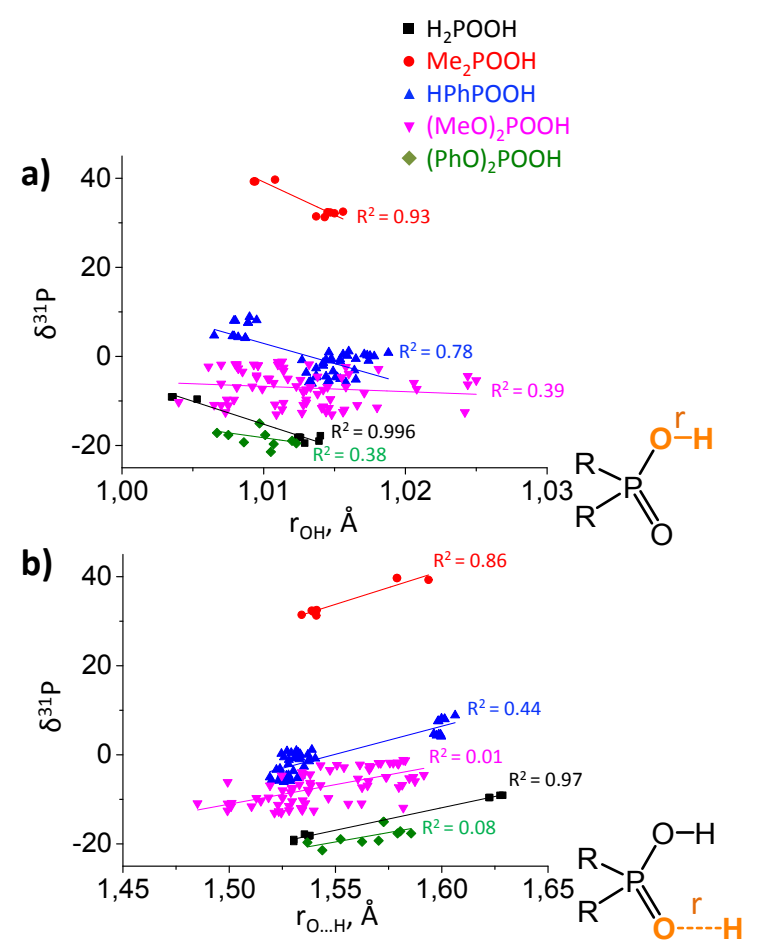

Figure S12. The dependence of the ${ }^{31} \mathrm{P}$ NMR chemical shift in cyclic dimers and cyclic trimers of acids 1-5 on (a) the $r_{\mathrm{OH}}$ distance and (b) the $r_{\mathrm{H} . . . \mathrm{O}}$ distance (see the definitions of these distances in the figure). 


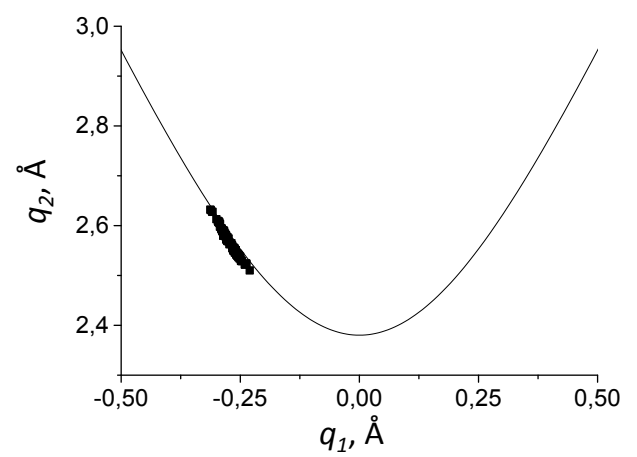

Figure S13. Interdependence of $q_{2}=r_{\mathrm{OH}}+r_{\mathrm{H} \cdots \mathrm{O}}$ and $q_{1}=1 / 2 \cdot\left(r_{\mathrm{OH}}-r_{\mathrm{H} \cdots \mathrm{O}}\right)$ distances for acids 1-5. The solid line corresponds to Equation 4 from [H.-H. Limbach et al., Israel J. Chem. 2009, 49, 199-216] (see also Figure $2 \mathrm{c}$ from the referenced paper). 


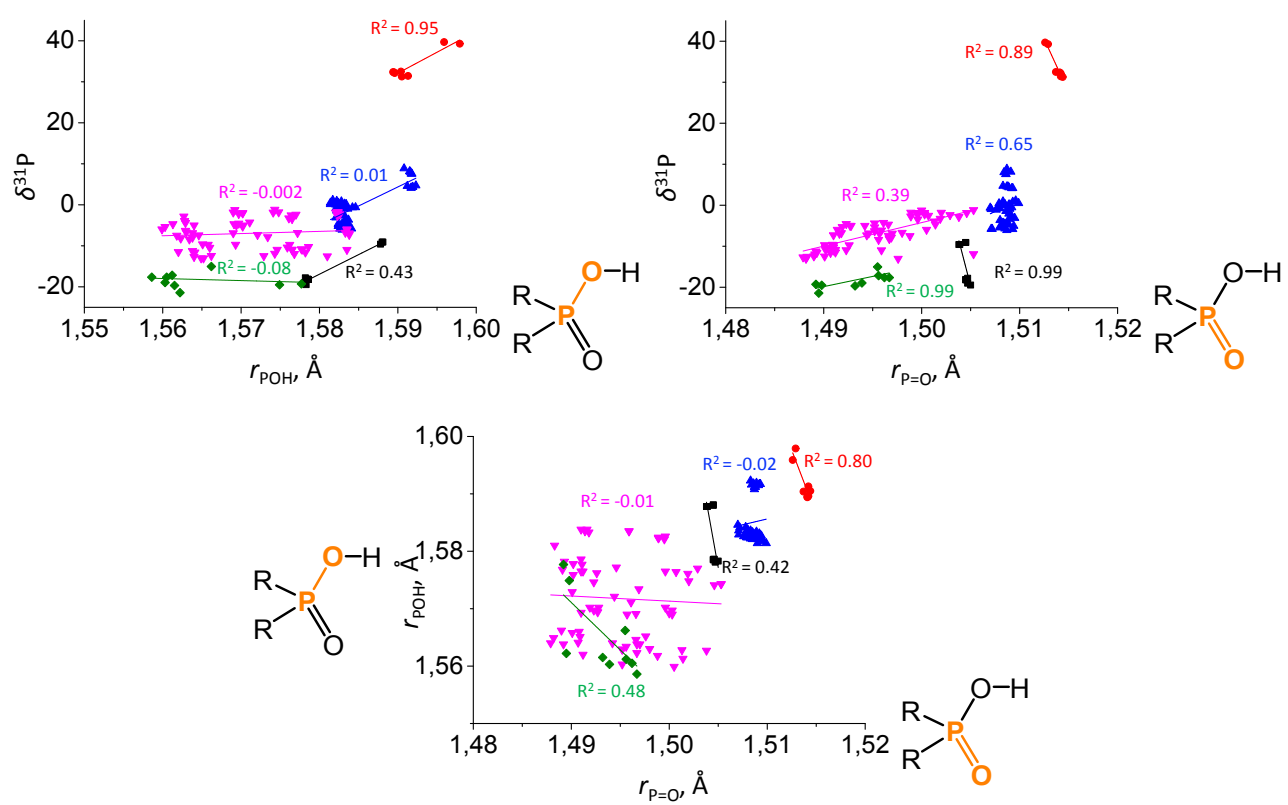

Figure S14. Interdependence of $\mathrm{P}-\mathrm{OH}$ and $\mathrm{P}=\mathrm{O}$ phosphorous-oxygen distances in cyclic dimers and cyclic trimers of acids $\mathbf{1}-\mathbf{5}$ and the sensitivity of $\delta \mathrm{P}$ values to these distances.. 